Article

\title{
Genotype-Epigenotype Interaction at the IGF2 DMR
}

\section{Susan K. Murphy ${ }^{1, *}$, Erin Erginer ${ }^{1}$, Zhiqing Huang ${ }^{1}$, Zachary Visco ${ }^{1}$ and Cathrine Hoyo ${ }^{2}$}

1 Department of Obstetrics and Gynecology, Division of Gynecologic Oncology, Duke University Medical Center, Box 91012, B223 LSRC Building, Durham, NC 27708, USA;

E-Mails: erin.erginer@gmail.com (E.E.); zhiqing.huang@duke.edu (Z.H.); zachary.visco@duke.edu (Z.V.)

2 Department of Biological Sciences, North Carolina State University, Raleigh, NC 27695, USA; E-Mail: choyo@ncsu.edu

* Author to whom correspondence should be addressed; E-Mail: susan.murphy@duke.edu; Tel.: +1-919-681-3423; Fax: +1-919-684-5336.

Academic Editor: J. Peter W. Young

Received: 4 May 2015 / Accepted: 21 August 2015 / Published: 28 August 2015

\begin{abstract}
Paternally expressed Insulin-like Growth Factor II (IGF2) encodes a gene whose protein product functions as a potent growth mitogen. Overexpression of IGF2 has been implicated in a wide number of disorders and diseases. IGF2 is regulated in part by differential methylation of the two parentally derived alleles. The differentially methylated region (DMR) located upstream of the imprinted promoters of $I G F 2$ exhibits plasticity under environmental stress and is hypomethylated in several types of cancer. Through bisulfite pyrosequencing and confirmation by nucleotide sequencing, we discovered a $\mathrm{CpG}$ to $\mathrm{CpC}$ transversion that results in hypomethylation of one of the three CpGs comprising this DMR. The presence of the polymorphism introduces a genetic rather than an environmentally-driven epigenetic source of hypomethylation that is additive to non-genetic sources.
\end{abstract}

Keywords: Insulin-like Growth Factor II; differentially methylated region; polymorphism; hypomethylation; $\mathrm{CpG}$ dinucleotide; imprinted gene 


\section{Introduction}

Numerous studies have focused on analysis of DNA methylation at a region located upstream of the three major imprinted promoters of IGF2 in humans. This region is one of the differentially methylated regions (DMRs) contributing to the regulation of Insulin-like Growth Factor II (IGF2), an imprinted gene that is expressed from the paternally-derived chromosome. IGF2 encodes for a small protein that is part of the insulin family and functions as a signaling molecule through binding to the IGF1 and insulin receptors [1]. IGF2 protein also binds to the membrane-bound and soluble forms of the IGF2 receptor, but this leads to internalization and degradation of IGF2 in the lysosomes. IGF2 has been shown to be deregulated in neurodevelopmental disorders [2-4], obesity and cancer [1,5], and is also known to have a critical role in memory consolidation in the brain [6-9].

DNA methylation occurs at the 5'-carbon position of the cytosine ring at cytosines that are followed by guanines (CG dinucleotides) in the DNA sequence. DNA methyltransferase (DNMT) enzymes recognize the palindromic 5'-CG-3' dinucleotide and are able to establish methylation de novo (DNMT3A and DNMT3B) or copy the methylation pattern of the parent DNA strand onto newly replicated DNA (DNMT1) (for review, please see [10,11]). Most CG dinucleotides throughout the genome are methylated. For genes subjected to parent-of-origin-dependent, monoallelic expression, CG dinucleotides are methylated on one parental strand and the same CG dinucleotide sequence on the other parental strand is unmethylated. This pattern of differential methylation is established during gametogenesis. For imprinted IGF2, these regulatory methylation marks are established during spermatogenesis, while the same sequences in the oocyte are unmethylated.

The multifaceted effects of IGF2 have led many, including our group, to study how the methylation status of this DMR, hereafter referred to as the IGF2 DMR, varies in disease states [12-15], as well as how methylation of this region is influenced by the in utero environment [16-23]. The first reports of methylation plasticity of the IGF2 DMR came from studies of individuals with colon cancer. In these studies, hypomethylation of the three $\mathrm{CpG}$ sites comprising the DMR was reported [24]. This was also detectable in peripheral blood of the individuals with colon cancer and in $10 \%$ of an otherwise healthy control population [25]. This same region has also been the focus of studies of individuals conceived during the Hunger Winter in the Netherlands at the end of World War II. Hypomethylation of this DMR was associated with exposure to caloric restriction in utero and this was evident decades later [26]. We have also found that methylation of this region in newborns is vulnerable to the effects of in utero exposure to cigarette smoking [18] and paternal obesity [27]. Hypomethylation has also been associated with increased circulating levels of IGF2 protein and increased birth weight [20], and conversely, low birth weight for children born to mothers who are depressed during pregnancy [17].

Herein we report the identification a $\mathrm{G}>\mathrm{C}$ polymorphism that directly affects one of the three $\mathrm{CpG}$ sites that comprise the IGF2 DMR, resulting in a $\mathrm{CpC}$ dinucleotide. The presence of this genetic polymorphism necessarily results in loss of methylation at this position, and thus overall decreased methylation of this DMR, through genetic ablation of the DNA methyltransferase recognition sequence at this $\mathrm{CpG}$ dinucleotide. Furthermore, the polymorphism appears to be more common in individuals with African heritage. These findings have implications for the methylation status of this region and consequent effects on IGF2 expression and imprinting. 


\section{Materials and Methods}

\section{Human Subjects}

Cervical Cancer Screening Cohort, Moshi, TZ: The study population has been previously described, along with eligibility criteria and recruitment procedures [28]. Briefly, 249 participants were recruited of 251 approached between November 2008 and March 2009 from the Reproductive Health Clinic (RHC) at Kilimanjaro Christian Medical Center (KCMC), a Cervical Cancer prevention clinic. Eligibility criteria included $\geq 18$ years of age, no prior history of an abnormal Pap test and willingness to participate. Invasive cervical cancer patients were also 18 years or older and were referred for colposcopic directed evaluations. Methylation data for the IGF2 DMR was generated for 166 of these women [13]. All subjects gave their informed consent for inclusion before they participated in this study. This study was conducted in accordance with the Declaration of Helsinki and the protocol was approved by the Research Ethics Board at KCMC and by the Duke University Institutional Review Board (Pro00008813).

The Newborn Epigenetics STudy (NEST), Durham, NC, USA: Study participants were enrolled between 2005-2009 and 2009-2011 as part of two multiethnic birth cohorts designed to identify the effects of early exposures on epigenetic profiles and phenotypic outcomes. These studies were conducted in accordance with the Declaration of Helsinki and approved by the Duke University Institutional Review Board (Pro00014548). Written consent was obtained from all mothers participating as study subjects prior to their inclusion in the study. Details regarding identification and enrollment procedures have been described elsewhere [19,29]. A total of 3646 pregnant women were approached and 2587 consented to participate in NEST. Methylation data for the IGF2 DMR was completed for 1104 newborns.

Bisulfite pyrosequencing. Genomic DNA was extracted from cervical scrapes, biopsies and invasive cervical cancer specimens (described in [30]) using Gentra Puregene Reagents (Qiagen, Valencia, CA, USA). Genomic DNA was extracted from umbilical cord blood in the NEST cohort also using Gentra Puregene reagents. The IGF2 DMR was analyzed by bisulfite pyrosequencing. Bisulfite modification of 800 ng genomic DNA was performed as previously described [31] or using the Zymo EZ DNA Methylation $^{\mathrm{TM}}$ Kit according to the manufacturer's recommendations (Zymo Research; Irvine, CA, USA). Pyrosequencing assays were designed using PSQ Assay Design Software and reactions were run on a Pyromark Q96 MD Pyrosequencer (Qiagen). The percent methylation for each of the CpGs within the target sequence was calculated using PyroQ CpG Software (Qiagen).

The region analyzed for the IGF2 DMR includes three $\mathrm{CpG}$ dinucleotides upstream of exon 3 (chr 11p15.5; CpG site 1: 2,169,519; CpG site 2: 2,169,516; and $\mathrm{CpG}$ site 3: 2,169,499; NCBI Human Genome Build 37/hg19) [24]. PCR and pyrosequencing primers and conditions were as described [17,19]. Assay validation was performed by analysis of defined ratios of plasmids that contain inserts derived from the bisulfite modified version of the methylated and unmethylated sequences, as previously described [32].

Nucleotide sequencing. Genomic DNA underwent PCR using primers F: 5'-TTT CCC TGG GAA TGC TCA TTC-3' and R: 5'-TTC TGT TGG ACA GGC TGC CC-3'. Genomic DNA (20 ng) was amplified in a $12.5 \mu \mathrm{L}$ PCR reaction volume using Hotstar Taq DNA polymerase (Qiagen). Thermocycler conditions were as follows: $95^{\circ} \mathrm{C}$ for 15 min followed by 35 cycles of $95{ }^{\circ} \mathrm{C}$ for $30 \mathrm{~s}, 67^{\circ} \mathrm{C}$ for $30 \mathrm{~s}$ and $72{ }^{\circ} \mathrm{C}$ for $30 \mathrm{~s}$, and a final extension at $72{ }^{\circ} \mathrm{C}$ for $10 \mathrm{~min}$. The amplicons were resolved on $2 \%$ agarose 
gels stained with ethidium bromide, the bands excised and amplicons separated from the agarose using GenElute Agarose Spin Columns (Sigma-Aldrich Corp.; St. Louis, MO, USA). Sequencing reactions were performed with BigDye Terminator reagents (Life Technologies; Grand Island, NY, USA). The product was sequenced using primer 5'-ATG CAT GAA GTT TTT CTC TG-3' at the Duke Genome Sequencing and Analysis Core Facility.

Statistical analysis. The potential over-representation of the polymorphism by race or by disease status was analyzed using Fisher's exact tests, with a $p$ value $<0.05$ considered significant.

\section{Results}

In prior published work, we have reported on methylation of the IGF2 DMR in two distinct cohorts. The first cohort analyzed included 1104 newborn participants of our Newborn Epigenetics Study from Durham, NC, using umbilical cord blood [17,19]. The second cohort analyzed were adult women from Moshi, Tanzania who provided cervical scrapes as part of their participation in a study of the progression of cervical intraepithelial neoplasia [28]. As part of our analysis of DNA methylation at regulatory regions of imprinted genes for these two studies, we used bisulfite pyrosequencing to quantify methylation of the same three $\mathrm{CpG}$ sites within the IGF2 DMR as first defined by Cui et al. [24]. We have previously validated the performance of this assay and shown the ability to discriminate $5 \%$ differences in methylation across the full range of possible values, using defined mixtures of methylated and unmethylated DNAs [17].

The pyrosequencing software uses information from a pre-defined sequence provided from user input during the assay setup that determines the order of nucleotides to be added, one at a time, during the sequencing reaction. The pre-defined sequence allows for detection of methylation status at $\mathrm{CpG}$ dinucleotides by step-wise injection of "T" (unmethylated) and then "C" (methylated). It then calculates the proportion of $\mathrm{C}$ from the peak height obtained relative to the total peak heights obtained for the $\mathrm{T}$ and $\mathrm{C}$ combined at that particular cytosine position, prior to injection of the guanine nucleotide at the following step that completes the $\mathrm{CpG}$ dinucleotide sequence. Mononucleotide runs, especially Ts, are common due to the conversion of non-CpG cytosines to uracils, then to thymines following PCR. Such runs are accounted for by the height of the peak obtained. The peak height should be divisible by the number of same nucleotides in the run to yield a number that is equivalent to that obtained for the peak height of a single nucleotide.

At the end of each run, the Pyromark CpG software assigns a "pass", "check quality" or "fail" descriptor for each sample. Whereas "pass" means that the sequence obtained and measured is within the limits of what might be expected given the sequence being analyzed. "Fail" means that a substantial problem has occurred except in the case of "no template" controls that are scored as "failed" runs when they have worked appropriately. A "check quality" score means there is a problem, but it may be minor or of no consequence and the software leaves it up to the user for interpretation. During study of the two cohorts described, we incidentally noted that there were multiple samples among our cohorts that were scored as "failed" runs by the PyroMark CpG software. We attempted to repeat the pyrosequencing reactions but to no avail. Since DNA quality can affect the ability to successfully perform pyrosequencing, we initially attributed failure of these runs to poor DNA quality and excluded these specimens from our original analyses. However, upon later visual inspection of the raw data, it was apparent that all of the "failed" runs were similar at the first CpG position of the IGF2 DMR and had a "failed" reference 
sequence at several positions downstream. These were followed by resumption of normal sequence patterns to the end of the sequence analyzed (Figure 1). The T incorporation at position 15 (Figure 1c) was much greater than expected, even if the cytosine had been unmethylated in the original sequence. This also led to problems with the second $\mathrm{CpG}$ site in the sequence (position 19), due to sequencing of the two alleles being out of sync until reaching an A residue (position 25) located a few bases prior to the 3rd CpG site (position 31), which shows the expected pattern of methylation in these individuals. We hypothesized that this pattern might result from the presence of a polymorphic nucleotide variant in the sequence.

Nucleotide sequencing of the unmodified genomic DNA indeed confirmed the presence of a G/C transversion polymorphism at $\mathrm{CpG}$ position 1 , which changes the $\mathrm{CpG}$ dinucleotide to a $\mathrm{CpC}$ dinucleotide on the affected allele (Figure 2). This SNP, subsequently identified as SNP rs116779517 (dbSNP 135), abolishes the 5'-CG-3' recognition sequence for the DNA methyltransferase enzymes - and thus the ability to methylate this cytosine. The decreased methylation observed at the second $\mathrm{CpG}$ dinucleotide was an artifact due entirely to the pyrosequencing method itself, whereby incorporation of nucleotides at the second $\mathrm{CpG}$ site occurred prematurely at the position of the first $\mathrm{CpG}$ site, rather than to any additional sequence variants being present, as described above.

We observed this polymorphism in 4.5\% (50/1104 individuals; minor allele frequency of 0.02 ) of our newborn cohort (Table 1) as well as in 11.4\% (19/166 individuals; minor allele frequency of 0.06 ) of our case-control study from Moshi, Tanzania (Table 2). The 1000 Genomes Project reports an overall minor allele frequency of 0.01 and 0.05 for individuals from West Africa [33]. All individuals from Tanzania in our study were of African descent, while 39 of the 50 newborns carrying the variant allele were from mothers who self-reported as African American (Fisher's exact test, $p<0.0001$ comparing African descent to non-African descent). These results indicate that this polymorphism may be significantly more prevalent in individuals with African heritage. However, the polymorphism was not enriched among individuals with cervical intraepithelial neoplasia (CIN; Fisher's exact test, $p=0.62$ ) or invasive cervical cancer (ICC; $p=0.39$ ). In addition, pyrosequencing of peripheral blood DNA from all nine individuals with CIN or ICC showed the presence of the polymorphism. We confirmed the polymorphism by Sanger sequencing of biopsy specimens for two patients with ICC and two patients with CIN; peripheral blood DNA from one patient with ICC and one with CIN was also sequenced with concordant results (data not shown). These results suggest that the presence of this polymorphism may not be associated with the onset or progression of cervical dysplasia, though larger studies are required for confirmation.

To confirm that the presence of the polymorphism influences the methylation status of CpG position 1 within the IGF2 DMR, we performed bisulfite sequencing of cloned alleles for six individuals carrying the variant allele (Figure 3). All six individuals showed a lack of methylation at $\mathrm{CpG}$ position 1 on the variant $T$ allele using this method. For three of the four cord blood specimens (UCB1-3 in Figure 3), the variant $\mathrm{T}$ allele was likely the paternally derived allele, since the other two $\mathrm{CpG}$ positions were nearly fully methylated across the clones analyzed. We are able to infer this because this region is normally differentially methylated, with methylation present on the paternally-derived allele [34,35]. For the two cervical biopsy specimens analyzed, more generalized hypomethylation at this DMR, a common finding in other gynecologic cancers [12,36], made it difficult to determine the parental origin of each allele. Regardless, these results demonstrate that the presence of the polymorphism prevents methylation at CpG position 1 by changing the CG DNA methyltransferase target sequence on the WT allele to a CC 
dinucleotide combination not recognized by these enzymes. Furthermore, if this polymorphism is present on the paternally-derived chromosome, this theoretically reduces the overall methylation at this locus from $\sim 50 \%$ to $\sim 34 \%$ in a diploid cell.

a
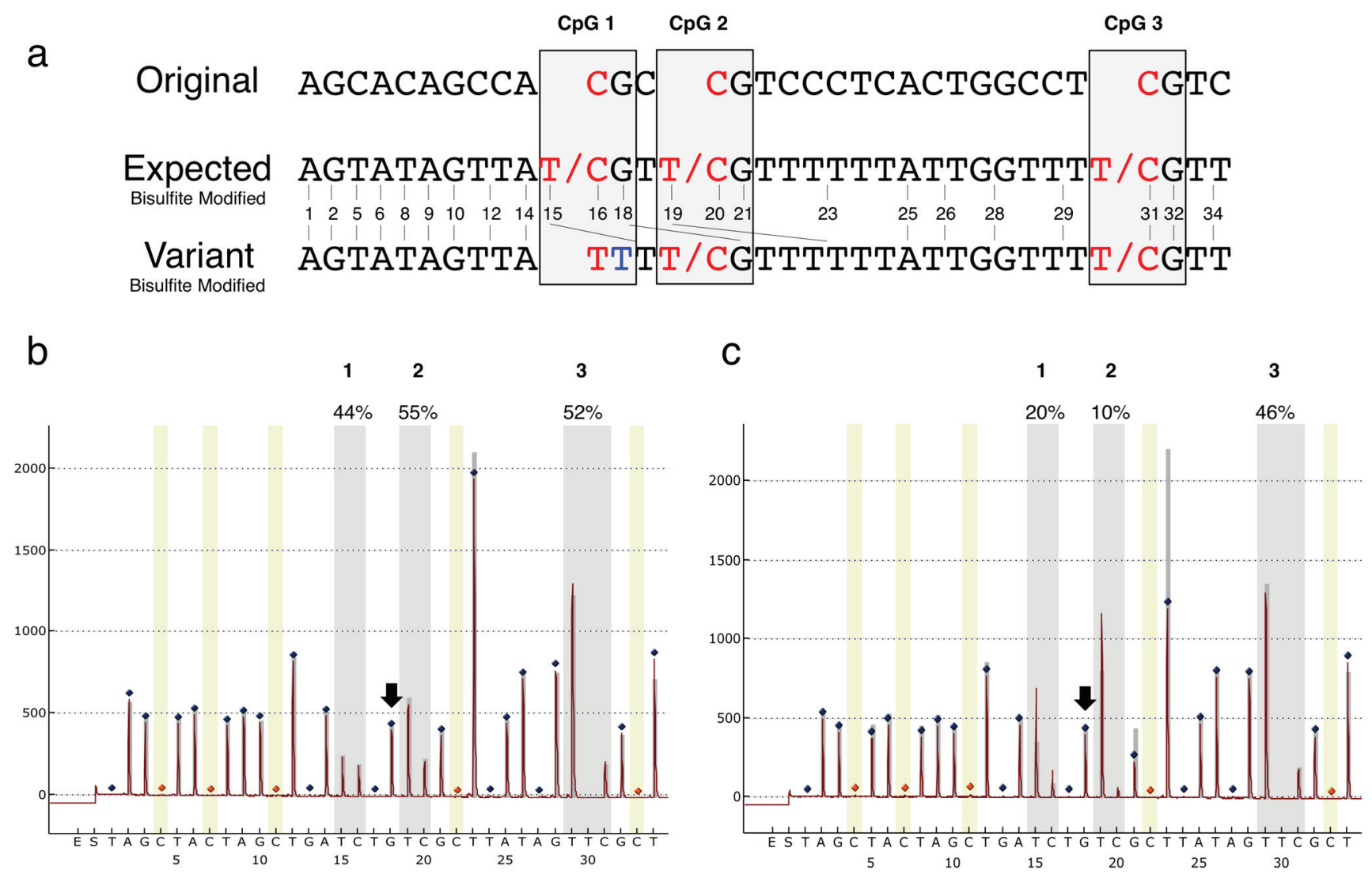

Figure 1. (a) The original sequence of interest for the IGF2 DMR region and the expected sequence from the bisulfite modified DNA and pyrosequencing, alongside the sequence for the variant allele. The three $\mathrm{CpG}$ dinucleotides are labeled; (b) Pyrogram obtained for an individual with wild type alleles; (c) Pyrogram from an individual with the variant allele, showing low methylation of $\mathrm{CpG}$ position 1 and a much higher than expected incorporation of T nucleotides at CpG 2 (position 19) due to the creation of a 3-4 base mononucleotide stretch of $\mathrm{T}$ residues. The number of $\mathrm{T}$ residues incorporated depends on the methylation status of the second $\mathrm{CpG}$ cytosine. This leads to premature incorporation of the downstream T nucleotides at position 19, early incorporation of the downstream $G$ at position 21 instead at position 18 on the variant allele and as consequence only half the anticipated $G$ height at the subsequent $\mathrm{G}$ at position 21, and lower than expected peak height for the Ts at position 23 since those on the variant allele had been incorporated at position 19. This also results in inaccuracy for the methylation status of the second $\mathrm{CpG}$ (site 20). The \% methylation measured is indicated above each $\mathrm{CpG}$ cytosine. $\mathrm{CpG}$ cytosine positions are indicated by the grey vertical bars. Blue diamonds, non-variable reference peaks; orange diamonds with yellow background bar, bisulfite treatment control and reference peak; narrow grey bars behind peaks, theoretical histogram for the sequence to analyze. Methylation data for the IGF2 DMR was generated for $n=166$ cervical cancer study specimens, of which $n=19$ had the variant allele, and $n=1104$ NEST cohort specimens, of which $n=50$ had the variant allele. 


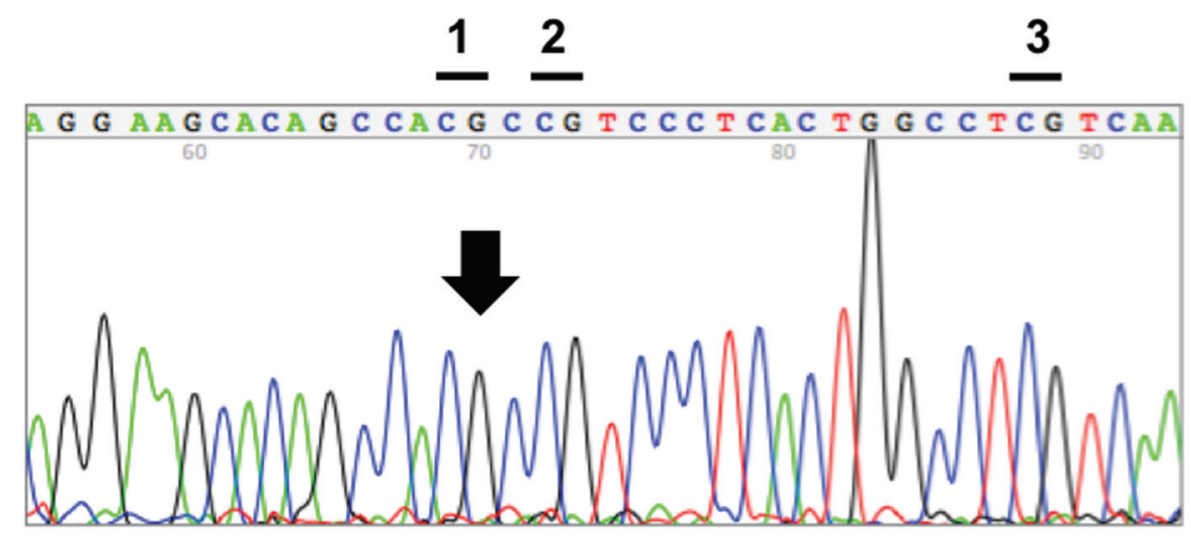

Variant

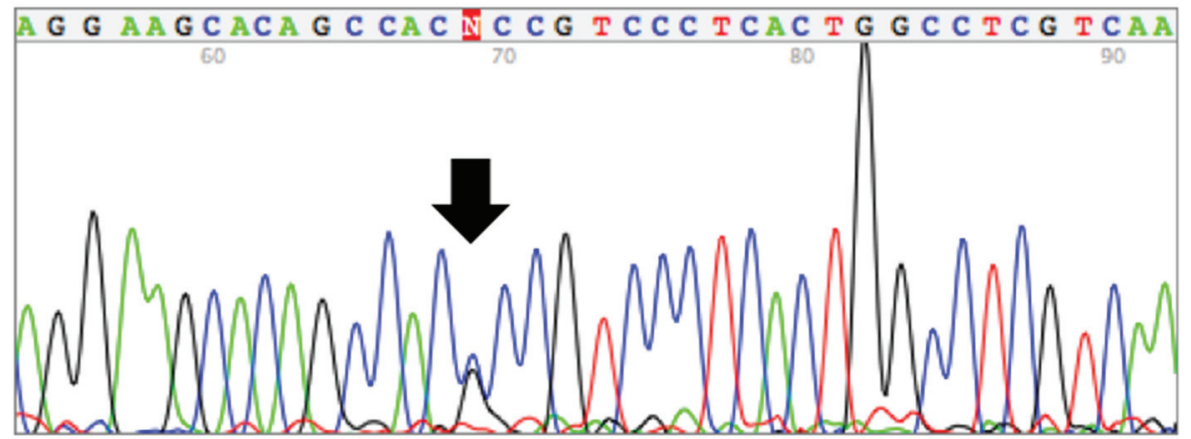

Figure 2. Representative nucleotide sequencing of the non-bisulfite modified genomic DNA for $n=14$ variant cervical tissue specimens, $n=2$ WT cervical cancer specimens and $n=6$ cord blood specimens. The non-variant (top) and variant (bottom) nucleotide sequences of the IGF2 DMR region are shown. The three $\mathrm{CpG}$ sites comprising this DMR are designated at the top and the position of SNP G $>$ C rs116779517 is indicated by the arrow.

Table 1. Newborn Epigenetics Study.

\begin{tabular}{lll}
\hline rs116779517 & WT (\%) & Heterozygote (\%) \\
\hline Caucasian & $510(99)$ & $7(1)$ \\
African American & $445(92)$ & $39(8)$ \\
Asian/Pacific Islander & $18(100)$ & $0(0)$ \\
Native American & $4(100)$ & $0(0)$ \\
Multiracial & $10(100)$ & $0(0)$ \\
Other & $53(96)$ & $2(4)$ \\
Don't Know & $5(100)$ & $0(0)$ \\
Missing & $9(82)$ & $2(18)$ \\
Total & $\mathbf{1 0 5 4}(\mathbf{9 5 )}$ & $\mathbf{5 0}(\mathbf{5})$ \\
\hline
\end{tabular}

Table 2. Tanzania Cervical Cancer Study.

\begin{tabular}{lll}
\hline rs116779517 & WT (\%) & Heterozygote (\%) \\
\hline Non-neoplastic & $88(90)$ & $10(10)$ \\
Neoplastic & $59(87)$ & $9(13)$ \\
Total & $\mathbf{1 4 7}(\mathbf{8 9})$ & $\mathbf{1 9}(\mathbf{1 1})$ \\
\hline
\end{tabular}




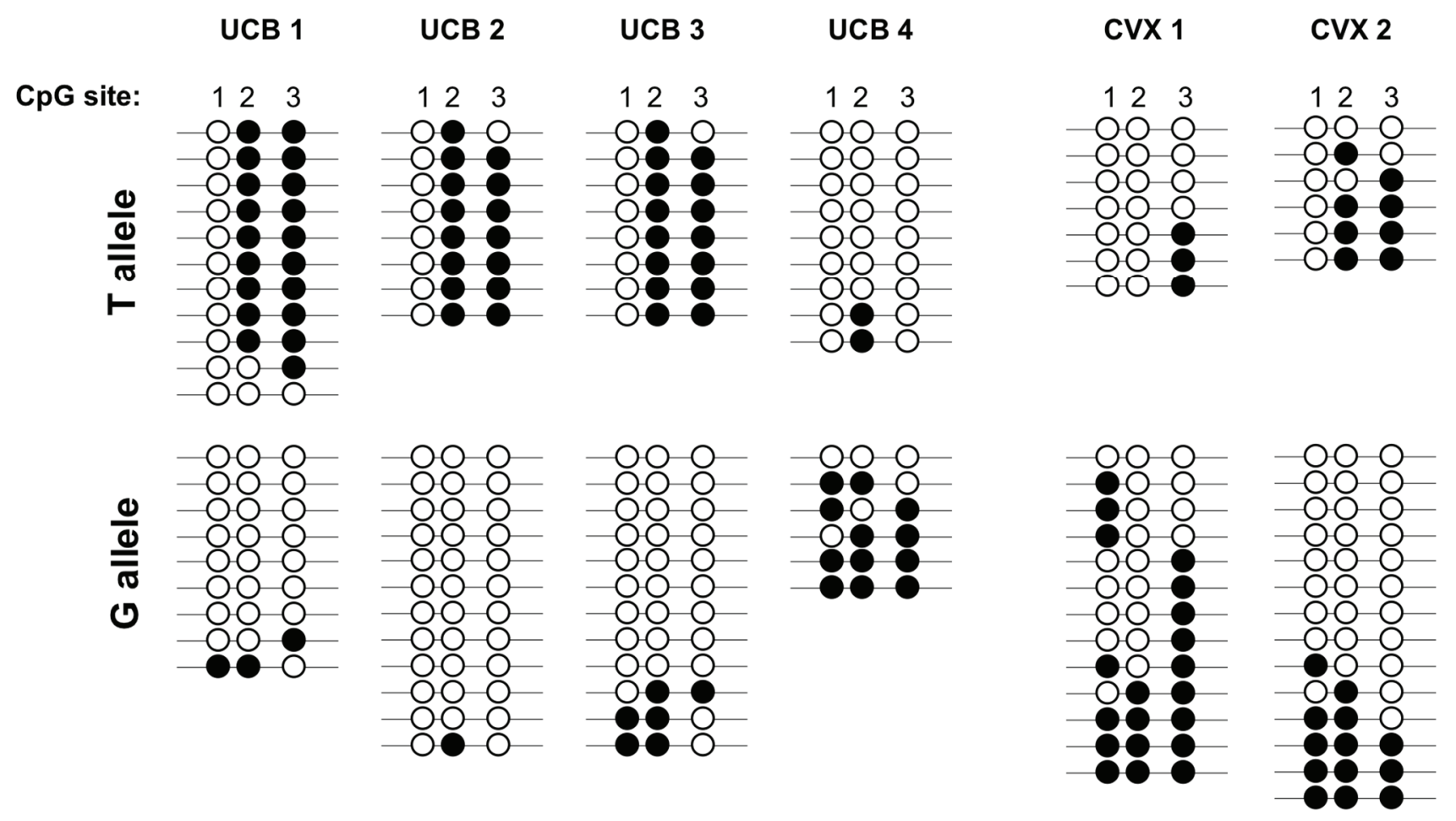

Figure 3. The variant allele lacks methylation at $\mathrm{CpG}$ position 1 of the IGF2 DMR. Bisulfite sequencing of cloned alleles for four umbilical cord blood DNA specimens (UCB1-UCB4) and two cervical biopsy specimens (CVX1-CVX2) from individuals carrying the variant allele. Each line represents a given clone and each circle represents one of the $\mathrm{CpG}$ dinucleotides within the IGF2 DMR. Filled circles indicate that the cytosine was methylated and the unfilled circles indicate that the cytosine was unmethylated within that clone. The data indicate that the variant $\mathrm{T}$ allele exhibits a lack of methylation at the first $\mathrm{CpG}$ site within the IGF2 DMR.

\section{Discussion}

The presence of this polymorphism is crucial to interpretation of any methylation studies of the IGF2 DMR, especially since the most common abnormality reported for this DMR in the literature is hypomethylation. The presence of this variant allele could go unnoticed using other bisulfite-based methods and lead to a finding of hypomethylation, assumed to be due to environmentally driven epigenetic variability rather than genetic variation. Indeed, we excluded the individuals carrying this variant in our own studies because these samples failed the pyrosequencing runs.

It has been known for some time that genetic and epigenetic variation together influence gene expression and disease susceptibility, however, specific empirical data are limited. Within the IGF2/H19 imprinted domain, Tobi et al. have reported that SNP rs2239681, upstream of the IGF2 DMR, is associated with decreased methylation [23], but this SNP exerts this effect over distance and does not directly alter $\mathrm{CpG}$ sites. A single nucleotide variant that alters methylation at a $\mathrm{CpG}$ site within one of the core binding sites for the CTCF insulator protein that regulates the IGF2/H19 imprinted domain has also been identified. The presence of the variant allele (rs10732516) affects genetically-induced hypomethylation, which is associated with higher birth weight [21]. In another study, Oertel et al. identified an A $>$ G SNP 
(rs1799971) that introduces a new CpG site in the OPRM1 gene; methylation at this site reduces expression and opioid receptor signaling [37]. Thus although an increasing number of studies are examining the cis activity of SNPs in relation to DNA methylation, there are also SNPs that directly affect methylation changes with a consequent change in phenotype that should also be further investigated.

Genetically predetermined hypomethylation may have substantial relevance to the expression of $I G F 2$ and risk of disease, perhaps more so than any environmentally-induced variation. We previously reported that a $1 \%$ change in methylation at this DMR was associated with a two-fold change in IGF2 transcription [18]. Others have reported that hypomethylation of these same CpGs is associated not only with loss of IGF2 imprinting, but also to risk of colon cancer, with hypomethylation detected in $10 \%$ of an otherwise unaffected population $[24,25,38]$. Race was not reported in these studies so it is unclear how this polymorphism might have contributed to the hypomethylation reported. Furthermore, the relevance of loss of imprinting is also uncertain, since other studies have detected loss of imprinting primarily where overall IGF2 expression is low [12,39]. We did not detect an association between the presence of the polymorphism and cervical neoplasia or cancer, however we examined a relatively small number of individuals. Nevertheless, if the prevalence of this polymorphic variant in individuals of African descent is confirmed by others, these findings could have substantial implications for colon cancer risk assessment in these individuals. Hypomethylation at this DMR may increase risk of cancer regardless of whether the hypomethylation is genetically and/or epigenetically determined.

We did not determine the parental origin of the rare variant allele. However, we have previously found that these $\mathrm{CpGs}$ are fully methylated in mature human spermatozoa [35], confirming reports of others $[34,40]$ and consistent with the methylation in somatic tissues being on the paternally-derived allele. Thus if the $\mathrm{G}>\mathrm{C}$ polymorphism is inherited from the father, it might be expected that there would be no methylation present at this $\mathrm{CpG}$ site. However, we did not observe $0 \%$ methylation at this $\mathrm{CpG}$ site in any of the samples analyzed that carry the nucleotide variant. The reason for this is evident when tracking the order of nucleotide incorporation in the pyrosequencing "sequence to analyze" and taking into account the pattern of methylation expected on the paternal allele. The sequence variant leads to additional " $\mathrm{T}$ " nucleotides incorporated at the first $\mathrm{CpG}$ site (i.e., the cytosine cannot be methylated in $\mathrm{CpC}$ context so both are converted by bisulfite, adding two $\mathrm{Ts}$ ); when the $\mathrm{C}$ is added for incorporation for the first $\mathrm{CpG}$ site, it is actually the second $\mathrm{CpG}$ site $\mathrm{C}$ nucleotide that ends up being incorporated at this position for the variant allele. Thus methylation at the first $\mathrm{CpG}$ site actually reflects that at the second $\mathrm{CpG}$ site. The second $\mathrm{CpG}$ site in turn exhibits very low methylation.

\section{Conclusions}

In conclusion, through bisulfite pyrosequencing and subsequent confirmation by nucleotide sequencing, we identified and validated a polymorphic variant that directly prevents methylation at one of the CpG dinucleotides that comprise the IGF2 DMR, a region for which hypomethylation has previously been associated with a compromised environment during prenatal development as well as risk of colon cancer. We found the variant in two distinct populations including one from Tanzania, Africa and the other from Durham, NC, USA. The variant is significantly more common in, but may not be exclusive to, individuals of African descent, and these findings indicate that epigenetic analysis of this region should take into account the potential genetic contribution to hypomethylation. 


\section{Acknowledgments}

We thank all of the study participants for their willingness to take part in this research. We are especially grateful for the contributions made by Francine Overcash, Rachel Maguire and Carole Grenier. This research was supported by NIH grants P30ES011961, R21ES015165, R01ES016772, R01DK085173, P01ES022831, P30AI064518 and R01CA1429983 and USEPA grant RD-83543701. The content is solely the responsibility of the authors and does not necessarily represent the official views of the NIH or the USEPA. Further, the NIH and USEPA do not endorse the purchase of any commercial products or services mentioned in the publication.

\section{Author Contributions}

Susan K. Murphy, Cathrine Hoyo, Erin Erginer and Zhiqing Huang conceived and designed the experiments; Erin Erginer, Zhiqing Huang and Zachary Visco performed the experiments; Susan K. Murphy analyzed the data and wrote the paper. All authors have read and approve of the manuscript.

\section{Conflicts of Interest}

The authors declare no conflict of interest.

\section{References}

1. Brouwer-Visser, J.; Huang, G.S. IGF2 signaling and regulation in cancer. Cytokine Growth Factor Rev. 2015, 26, 371-377.

2. Bai, B.; Zhang, Q.; Liu, X.; Miao, C.; Shangguan, S.; Bao, Y.; Guo, J.; Wang, L.; Zhang, T.; Li, H.; et al. Different epigenetic alterations are associated with abnormal IGF2/IGF2 upregulation in neural tube defects. PLOS ONE 2014, 9, e113308.

3. Bracko, O.; Singer, T.; Aigner, S.; Knobloch, M.; Winner, B.; Ray, J.; Clemenson, G.D., Jr.; Suh, H.; Couillard-Despres, S.; Aigner, L.; et al. Gene expression profiling of neural stem cells and their neuronal progeny reveals IGF2 as a regulator of adult hippocampal neurogenesis. $J$. Neurosci. 2012, 32, 3376-3387.

4. Wu, L.; Wang, L.; Shangguan, S.; Chang, S.; Wang, Z.; Lu, X.; Zhang, Q.; Wang, J.; Zhao, H.; Wang, F.; et al. Altered methylation of IGF2 DMR0 is associated with neural tube defects. Mol. Cell. Biochem. 2013, 380, 33-42.

5. Livingstone, C. IGF2 and cancer. Endocr. Relat. Cancer 2013, 20, R321-R339.

6. Alberini, C.M.; Chen, D.Y. Memory enhancement: consolidation, reconsolidation and insulin-like growth factor 2. Trends Neurosci. 2012, 35, 274-283.

7. Iwamoto, T.; Ouchi, Y. Emerging evidence of insulin-like growth factor 2 as a memory enhancer: a unique animal model of cognitive dysfunction with impaired adult neurogenesis. Rev. Neurosci. 2014, 25, 559-574.

8. Pascual-Lucas, M.; Viana da Silva, S.; di Scala, M.; Garcia-Barroso, C.; Gonzalez-Aseguinolaza, G.; Mulle, C.; Alberini, C.M.; Cuadrado-Tejedor, M.; Garcia-Osta, A. Insulin-like growth factor 2 reverses memory and synaptic deficits in APP transgenic mice. EMBO Mol. Med. 2014, 6, 1246-1262. 
9. Wang, X.; Wang, L.P.; Tang, H.; Shan, W.Y.; Wang, X.; Liu, D.; Wu, Y.Y.; Tian, Q.; Wang, J.Z.; Zhu, L.Q.; et al. Acetyl-L-carnitine rescues scopolamine-induced memory deficits by restoring insulin-like growth factor II via decreasing p53 oxidation. Neuropharmacology 2014, 76, 80-87.

10. Cooper, D.N. Eukaryotic DNA methylation. Hum. Genet. 1983, 64, 315-333.

11. Jurkowska, R.Z.; Jurkowski, T.P.; Jeltsch, A. Structure and function of mammalian DNA methyltransferases. Chembiochem 2011, 12, 206-222.

12. Murphy, S.K.; Huang, Z.; Wen, Y.; Spillman, M.A.; Whitaker, R.S.; Simel, L.R.; Nichols, T.D.; Marks, J.R.; Berchuck, A. Frequent IGF2/H19 domain epigenetic alterations and elevated IGF2 expression in epithelial ovarian cancer. Mol. Cancer Res. 2006, 4, 283-292.

13. Vidal, A.C.; Henry, N.M.; Murphy, S.K.; Oneko, O.; Nye, M.; Bartlett, J.A.; Overcash, F.; Huang, Z.; Wang, F.; Mlay, P.; et al. PEG1/MEST and IGF2 DNA methylation in CIN and in cervical cancer. Clin. Transl. Oncol. 2014, 16, 266-272.

14. Rumbajan, J.M.; Maeda, T.; Souzaki, R.; Mitsui, K.; Higashimoto, K.; Nakabayashi, K.; Yatsuki, H.; Nishioka, K.; Harada, R.; Aoki, S.; et al. Comprehensive analyses of imprinted differentially methylated regions reveal epigenetic and genetic characteristics in hepatoblastoma. BMC Cancer 2013, doi:10.1186/1471-2407-13-608.

15. Baba, Y.; Nosho, K.; Shima, K.; Huttenhower, C.; Tanaka, N.; Hazra, A.; Giovannucci, E.L.; Fuchs, C.S.; Ogino, S. Hypomethylation of the IGF2 DMR in colorectal tumors, detected by bisulfite pyrosequencing, is associated with poor prognosis. Gastroenterology 2010, 139, 1855-1864.

16. Perkins, E.; Murphy, S.K.; Murtha, A.P.; Schildkraut, J.; Jirtle, R.L.; Demark-Wahnefried, W.; Forman, M.R.; Kurtzberg, J.; Overcash, F.; Huang, Z.; et al. Insulin-like Growth Factor 2/H19 methylation at birth and risk of overweight and obesity in children. J. Pediatr. 2012, 161, 31-39.

17. Liu, Y.; Murphy, S.K.; Murtha, A.P.; Fuemmeler, B.F.; Schildkraut, J.; Huang, Z.; Overcash, F.; Kurtzberg, J.; Jirtle, R.; Iversen, E.S.; et al. Depression in pregnancy, infant birth weight and DNA methylation of imprint regulatory elements. Epigenetics 2012, 7, 735-746.

18. Murphy, S.K.; Adigun, A.; Huang, Z.; Overcash, F.; Wang, F.; Jirtle, R.L.; Schildkraut, J.M.; Murtha, A.P.; Iversen, E.S.; Hoyo, C. Gender-specific methylation differences in relation to prenatal exposure to cigarette smoke. Gene 2012, 494, 36-43.

19. Hoyo, C.; Murtha, A.P.; Schildkraut, J.M.; Forman, M.R.; Calingaert, B.; Demark-Wahnefried, W.; Kurtzberg, J.; Jirtle, R.L.; Murphy, S.K. Folic acid supplementation before and during pregnancy in the Newborn Epigenetics STudy (NEST). BMC Public Health 2011, doi:10.1186/1471-2458-11-46.

20. Hoyo, C.; Fortner, K.; Murtha, A.P.; Schildkraut, J.M.; Soubry, A.; Demark-Wahnefried, W.; Jirtle, R.L.; Kurtzberg, J.; Forman, M.R.; Overcash, F.; et al. Association of cord blood methylation fractions at imprinted insulin-like growth factor 2 (IGF2), plasma IGF2, and birth weight. Cancer Causes Control 2012, 23, 635-645.

21. Hernandez-Valero, M.A.; Rother, J.; Gorlov, I.; Frazier, M.; Gorlova, O.Y. Interplay between polymorphisms and methylation in the H19/IGF2 gene region may contribute to obesity in Mexican-American children. J. Dev. Orig. Health Dis. 2013, 4, 499-506.

22. St-Pierre, J.; Hivert, M.F.; Perron, P.; Poirier, P.; Guay, S.P.; Brisson, D.; Bouchard, L. IGF2 DNA methylation is a modulator of newborn's fetal growth and development. Epigenetics 2012, 7, 1125-1132. 
23. Tobi, E.W.; Slagboom, P.E.; van Dongen, J.; Kremer, D.; Stein, A.D.; Putter, H.; Heijmans, B.T.; Lumey, L.H. Prenatal famine and genetic variation are independently and additively associated with DNA methylation at regulatory loci within IGF2/H19. PLoS ONE 2012, 7, e37933.

24. Cui, H.; Onyango, P.; Brandenburg, S.; Wu, Y.; Hsieh, C.L.; Feinberg, A.P. Loss of imprinting in colorectal cancer linked to hypomethylation of H19 and IGF2. Cancer Res. 2002, 62, 6442-6446.

25. Cui, H.; Cruz-Correa, M.; Giardiello, F.M.; Hutcheon, D.F.; Kafonek, D.R.; Brandenburg, S.; Wu, Y.; He, X.; Powe, N.R.; Feinberg, A.P. Loss of IGF2 imprinting: A potential marker of colorectal cancer risk. Science 2003, 299, 1753-1755.

26. Heijmans, B.T.; Tobi, E.W.; Stein, A.D.; Putter, H.; Blauw, G.J.; Susser, E.S.; Slagboom, P.E.; Lumey, L.H. Persistent epigenetic differences associated with prenatal exposure to famine in humans. Proc. Natl. Acad. Sci. USA 2008, 105, 17046-17049.

27. Soubry, A.; Schildkraut, J.M.; Murtha, A.; Wang, F.; Huang, Z.; Bernal, A.; Kurtzberg, J.; Jirtle, R.L.; Murphy, S.K.; Hoyo, C. Paternal obesity is associated with IGF2 hypomethylation in newborns: results from a Newborn Epigenetics Study (NEST) cohort. BMC Med. 2013, doi:10.1186/1741-7015-11-29.

28. Vidal, A.C.; Murphy, S.K.; Hernandez, B.Y.; Vasquez, B.; Bartlett, J.A.; Oneko, O.; Mlay, P.; Obure, J.; Overcash, F.; Smith, J.S.; et al. Distribution of HPV genotypes in cervical intraepithelial lesions and cervical cancer in Tanzanian women. Infect. Agent Cancer 2011, doi:10.1186/1750-9378-6-20.

29. Liu, Y.; Hoyo, C.; Murphy, S.K.; Huang, Z.; Overcash, F.; Thompson, J.; Brown, H.; Murtha, A.P. DNA methylation at imprint regulatory regions in preterm birth and infection. Am. J. Obstet. Gynecol. 2013, 208, 395.e1-395.e7.

30. Nye, M.D.; Hoyo, C.; Huang, Z.; Vidal, A.C.; Wang, F.; Overcash, F.; Smith, J.S.; Vasquez, B.; Hernandez, B.; Swai, B.; et al. Associations between methylation of paternally expressed gene 3 (PEG3), cervical intraepithelial neoplasia and invasive cervical cancer. PLOS ONE 2013, 8, e56325.

31. Huang, Z.; Wen, Y.; Shandilya, R.; Marks, J.R.; Berchuck, A.; Murphy, S.K. High throughput detection of M6P/IGF2R intronic hypermethylation and LOH in ovarian cancer. Nucleic Acids Res. 2006, 34, 555-563.

32. Wong, H.L.; Byun, H.M.; Kwan, J.M.; Campan, M.; Ingles, S.A.; Laird, P.W.; Yang, A.S. Rapid and quantitative method of allele-specific DNA methylation analysis. Biotechniques 2006, 41, 734-739.

33. Genomes Project Consortium; Abecasis, G.R.; Altshuler, D.; Auton, A.; Brooks, L.D.; Durbin, R.M.; Gibbs, R.A.; Hurles, M.E.; McVean, G.A. A map of human genome variation from population-scale sequencing. Nature 2010, 467, 1061-1073.

34. Murrell, A.; Ito, Y.; Verde, G.; Huddleston, J.; Woodfine, K.; Silengo, M.C.; Spreafico, F.; Perotti, D.; de Crescenzo, A.; Sparago, A.; et al. Distinct methylation changes at the IGF2-H19 locus in congenital growth disorders and cancer. PLOS ONE 2008, 3, e1849.

35. Huang, Z.; Murphy, S.K. Increased Intragenic IGF2 Methylation is associated with repression of insulator activity and elevated expression in serous ovarian carcinoma. Front. Oncol. 2013, doi:10.3389/fonc.2013.00131. 
36. Dammann, R.H.; Kirsch, S.; Schagdarsurengin, U.; Dansranjavin, T.; Gradhand, E.; Schmitt, W.D.; Hauptmann, S. Frequent aberrant methylation of the imprinted IGF2/H19 locus and LINE1 hypomethylation in ovarian carcinoma. Int. J. Oncol. 2010, 36, 171-179.

37. Oertel, B.G.; Doehring, A.; Roskam, B.; Kettner, M.; Hackmann, N.; Ferreiros, N.; Schmidt, P.H.; Lotsch, J. Genetic-epigenetic interaction modulates mu-opioid receptor regulation. Hum. Mol. Genet. 2012, 21, 4751-4760.

38. Cui, H. Loss of imprinting of IGF2 as an epigenetic marker for the risk of human cancer. Dis. Markers 2007, 23, 105-112.

39. Waterland, R.A.; Lin, J.R.; Smith, C.A.; Jirtle, R.L. Post-weaning diet affects genomic imprinting at the insulin-like growth factor 2 (Igf2) locus. Hum. Mol. Genet. 2006, 15, 705-716.

40. Boissonnas, C.C.; Abdalaoui, H.E.; Haelewyn, V.; Fauque, P.; Dupont, J.M.; Gut, I.; Vaiman, D.; Jouannet, P.; Tost, J.; Jammes, H. Specific epigenetic alterations of IGF2-H19 locus in spermatozoa from infertile men. Eur. J. Hum. Genet. 2010, 18, 73-80.

(C) 2015 by the authors; licensee MDPI, Basel, Switzerland. This article is an open access article distributed under the terms and conditions of the Creative Commons Attribution license (http://creativecommons.org/licenses/by/4.0/). 